\title{
AC 2012-3387: ENHANCING CROSS-CULTURAL INTERACTION IN COURSES WITH A LARGE COMPONENT OF VISITING STUDY ABROAD STU- DENTS
}

\section{Dr. Alex Friess, Rochester Institute of Technology, Dubai}

\begin{abstract}
Alex Friess holds a Ph.D. in aeronautical engineering and a B.Sc. in physics from Rensselaer Polytechnic Institute (Troy, N.Y. 1997), and has served as Associate Professor of mechanical engineering at RIT, Dubai, since 2009. In addition to undergraduate activities, he teaches in the sustainable energy concentration of RIT, Dubai's mechanical engineering master's program. Friess' industrial and academic career spans a variety of consulting and entrepreneurial activities in Europe, Asia, and Africa, most notably as founding faculty in mechanical engineering at Dubai Aerospace Enterprise University and as Co-founder and CTO of RFB Solartech SL, a company that specializes in solar thermal solutions for the Spanish residential and commercial sector. Friess has established and leads the Sustainable Energy and Energy Efficiency Research Group at RIT, Dubai. Current research interests are focused on sustainability and energy efficiency and the field of engineering design and design education, with a focus on multinational student collaboration and educating the "global engineer."
\end{abstract}

\section{Dr. Ivan E. Esparragoza, Pennsylvania State University, Brandywine}

Ivan E. Esparragoza is an Associate Professor of engineering at Penn State Brandywine. His interests are in engineering design education, innovative design, global design, and global engineering education. He has introduced multinational design projects in a freshman introductory engineering design course in collaboration with institutions in Latin America and the Caribbean as part of his effort to contribute to the formation of world class engineers for the Americas. He is actively involved in the International Division of the American Society for Engineering Education, in the Latin American and Caribbean Consortium of Engineering Institution (LACCEI) as Vice-President for Research, and in the International D+Federation of Engineering Education Societies as member of the Executive Board.

\section{Dylan Connole}




\title{
Enhancing cross-cultural interaction in courses with a large component of visiting study-abroad students
}

\begin{abstract}
International student mobility programs enhance the global exposure of students, and represent a vital and increasing component in the offering of many universities worldwide. These programs often involve the travel of a group of students to an overseas location, where they, in addition to a variety of cultural activities, also register for a series of courses towards their degree completion. These courses are taken jointly with their local peers. While collaborative exchange programs among different universities often target individual student mobility, and the travelling student (or small group of students) generally represents a minority in the overseas class, in the case of branch campuses, and due to the seamless integration of the academic coursework and perhaps more systematic organization of the study abroad experience, an increasingly homogeneous and larger travelling student group can result, which may in turn imply that the visiting students can represent a large fraction of a class. This more even distribution between study abroad and local students can generate classroom dynamics that effectively split the class into two groups, and thus are not supportive of the cross-cultural interaction dimension of the international experience.
\end{abstract}

This work discusses the observed barriers to cross-cultural collaboration in the classroom at the Dubai campus of Rochester Institute of Technology (RTI), where this effect has been noted in a senior/graduate level course in renewable energy systems that was composed by over $50 \%$ of study-abroad students from the main campus. Class dynamics of preferably working with peers from the same background developed, in part due to an easier out-of-class access to these peers, and in part due to a higher comfort level in the peer interaction. While the academic learning outcomes were met by both groups, this experience has shown that in order to increase cross-cultural interaction in the classroom, specific learning criteria and outcomes that stress global competencies need to be introduced. This paper presents the lessons learned in the process (including out of classroom factors that affect in classroom collaboration), and presents a work in progress of designing appropriate learning objectives, activities and assessment tools to foster development of global competencies in classes with a large and homogeneous component of study abroad students.

\section{Introduction}

Over the past two decades, and in light of the growing globalization of industry and business, global competencies have evolved into an important component in university level education. In particular engineering, with its traditionally lesser focus on process skills, has increased awareness of the importance of these skills in a global and international environment. Through the introduction of the 2000 ABET criteria, this need has been further recognized, and integrated as one of the basic outcomes of any engineering program ${ }^{1}$. In 2004 the National Academies published The Engineer of $2020^{2}$, followed in 2005 by Educating the Engineer of $2020^{3}$. Later, the National Academies was asked by representatives of the U.S. Senate and House of Representatives to formulate strategies that policymakers could propose so the U.S. can successfully compete, prosper, and secure the global community of the $21^{\text {st }}$ century. The resulting report, Rising above the Gathering Storm ${ }^{4}$ was published in 2006 . That year, Continental Corporation ${ }^{5}$ funded the first scientific global engineering study conducted by eight prestigious universities around the world ${ }^{5}$. The study resulted in four recommendations: (1) A key qualification of engineering graduates must be global 
competence; (2) transnational mobility for engineering students, researchers, and professionals needs to become a priority; (3) global engineering excellence critically depends on a partnerships, especially those that link engineering education to professional practice; and (4) research is urgently needed on engineering in a global context.

The challenges of educating world class engineers with global competencies have generated changes in the paradigm of engineering education that explore great variety of teaching/learning approaches with the aim of forming the future engineers with a set of professional skills to be productive and competitive in the global economy. Those approaches involve different initiatives including online collaboration, multinational projects, and semester or yearlong study abroad experiences among the most common ones ${ }^{6,7}$.

As a result of the need of forming engineers with global competencies, RIT has been very active in creating international opportunities for their students. In order to facilitate this international experience, RIT, and through its global campuses initiative, has established a series of global branch campuses and programs in Kosovo, Croatia, the Dominican Republic, and finally Dubai. The United Arab Emirates (UAE) campus is the largest one, and the only one that offers undergraduate engineering programs. While still ramping up its operation, RIT Dubai has already been very successful in attracting study abroad students from the main campus. This paper discusses the observed barriers to cross-cultural collaboration in the classroom at the Dubai campus of RTI, where this effect has been noted in a senior/graduate level course composed by over $50 \%$ of study-abroad students from the main US campus. Faculty and students observations about the class interaction are presented and some recommendations are formulated to enhance the cross cultural experience with the aim to facilitate the development of global competencies in engineering students.

\section{Rationale}

The need of educating engineers not only with solid technical knowledge but also with a set of professional skills including global competencies has been understood by the academia. What might be still a topic of discussion is the definition of global competencies. From the engineering perspective, it can be argued that global competencies are a set of knowledge and skills that allow people to work effectively with others that think and act differently due to cultural differences. Other definitions include a shift in perception from an ethno-centric standpoint, where the own culture and environment assumes a central role, to an ethnorelative standpoint, where the own position is seen as a simple constituent of a global environment ${ }^{8}$. Warnick ${ }^{9}$ compiled information and suggested the set of global competencies necessaries for engineers to succeed in a global environment and those attributes are summarized in Table 1:

Table 1: Common Global Competencies

\begin{tabular}{|l|l|}
\hline 1 & Exhibit global mindset \\
\hline 2 & Appreciate and understand different cultures \\
\hline 3 & Demonstrate world and local knowledge \\
\hline 4 & Communicate cross-culturally \\
\hline 5 & Speak more than one language including English \\
\hline 6 & Understand international business, law and technical elements \\
\hline 7 & Live and work in transnational engineering environment \\
\hline 8 & Work in international teams \\
\hline
\end{tabular}


From the list of skills summarized in Table 1, it is evident that developing those abilities while studying engineering requires an approach beyond the lecture in the classroom and the traditional assignments ${ }^{10}$. There is a need of building cross-cultural skills by facilitating learning experiences that complement the engineering curriculum. International experiences are a mechanism to start building cross-cultural awareness. These international experiences range from online interaction (courses, seminars, short collaborative projects, etc.) to travel abroad experiences (short trips, semester long or yearlong study, research or internship experiences); however, merely travelling abroad, while ensuring an international experience, does not guarantee the development of global competencies. It is necessary to get immersed in another culture to develop a deep understanding of cultural differences and empathy for other cultures. This will promote acceptance and tolerance, facilitating group interaction and

discussion. Cross cultural experiences are also important in developing global competencies because they allow getting acquainted with social behavior and forms of communication in different cultures ${ }^{11}$. Therefore, an effective educational travel abroad experience should: (a) facilitate depth understanding of cultural differences; (b) promote empathy for a different culture; (c) facilitate cross-cultural communication and interaction; (d) provide transnational experience (live and work); and (e) promote multi-cultural teamwork.

Of particular interest in this paper is the quarter or semester long study abroad experience as a means of facilitating the development of global competencies. This work was motivated by the observed classroom dynamics in a senior undergraduate and first year graduate level engineering course on Renewable Energy Systems held at RIT Dubai during the winter quarter 2010-11.

\section{The course}

The course Renewable Energy Systems discusses a variety of applications of a range of renewable energy technologies, and synthesizes this material through a term project composed of both collaborative and individual portions. While the delivery is primarily lecture-style, the projects require both individual and team presentations, and some class time was devoted for the students to interact with their team members. However, the team composition was open; the students were free to choose their respective teammates. The reasoning behind this free choice option was to enable the students to choose appropriate subject areas that are aligned with senior projects that were running in parallel. In addition, in previous editions of this course (with no study abroad students present), no irregularities in the class dynamics were observed, and thus the need to address the issues reported here had not been observed previously.

The students in the class were composed of a group of six local UAE students (who originate from the Middle East and North Africa countries), and a group of nine US students visiting on a study abroad trip from the main campus in Rochester, NY. This international composition required a more international perspective on the topics presented. While for UAE students it is most important to address solar based technologies (and perhaps less emphasis on regionally less relevant technologies, such as hydroelectric), in this case the content had to be adapted to include renewable energy (RE) technologies typically encountered in the US, such as wind energy. However, a focus on solar technologies (concentrating and non-concentrating solar thermal and Photovoltaic (PV)) was still maintained. 
The project component of the course grade (30\%) required the students to write a program that would calculate the solar position and resulting irradiance on an arbitrarily oriented surface (taking into account both geometric and atmospheric attenuation effects on incident irradiance). This instantaneous irradiance was then integrated hourly over the year to simulate the overall incident energy. The second portion of the project then proceeded to utilize this program to size and preliminarily design $100 \mathrm{MW}$ concentrating solar thermal and PV utility scale power plants. The overall project grade was split $60 \%$ towards developing the program that calculates energy collected on an arbitrarily oriented surface in a year, this portion being an individual assignment, and $40 \%$ on the teamwork portion, which consisted in the design and sizing of the utility scale electric plants. The teams were composed of 2 to 3 people, and composed at will. The team assignments took place early in the term, and while the natural consequence of the students choosing their friends to be in their team was noted, the consequence of this choice in heavily impairing any cross cultural communication within the course was not realized. As this became more obvious, it was already too late to reassign students to other teams, as this would have hampered their progress towards achieving the team objectives.

The reduced size of the class undoubtedly introduced a large uncertainty in the statistical data. For this reason the quantitative survey results presented later should not be viewed as conclusive and only assumes a supportive role to the in-class observations. However, as will be seen, the correlation between both sets of data is good, and thus reinforces the conclusions taken from this experience. The lessons learned and proposed solutions presented here are intended to provide a starting point for future implementations of the course, and thus this work should be viewed as work-in-progress.

\section{Faculty Observations}

While in the initial phases of the course the normal tendency of the students to sit with their friends was observed, this group-forming tendency was assumed to decrease as the students become more familiar with each other, especially in a relatively small class such as this one. However, during the course of the term, this tendency to concentrate interaction to the familiar group was observed to increase. The following specific in-class observations were made throughout the semester:

- Physical separation: the classroom used was standard, with a central isle to access to the desks located on both sides. The students' tendency was to occupy the sides according to their origin: the study abroad students would cluster together, and the different nationalities (primarily Iranian and Indian) of the local graduate students would also sit together. Often the study abroad group would choose one side of the class, and the local students the other.

- In-class interaction: Upon discussion or answering questions, the students within each group would tend to interact primarily among themselves. That is, generally study abroad students would comment / interact with their peers in answering a question, but not (or to a much lesser degree) with the local students, and vice versa. If the interaction took place, on occasion it even assumed a certain "rivalry" tone.

- Interaction during idle times: At the beginning and end of each class and during occasional idle time, very rarely any discussion between the integrands of the different groups was noted, and if so only regarding very specific assignments. Verbal 
communication shifted from being relaxed and amicable internally, to more tense and factual among the groups. While this is often the case if language barriers exist, in this case, although the local students were all non-native English speakers, their level of proficiency was such that there were no language related communication barriers.

- Out of class opportunities to mingle: The study abroad students all resided at the student housing complex, with is located relatively far from the campus (bus ride is required). In addition, the local graduate students were often working professionals, which means that they did not spend much time on campus during daytime and primarily only attended for their night classes.

- Differing age groups: The course catered for both upper-class undergraduates and first year graduate students. The visiting study abroad group constituted the undergraduate group, while the local students were all first year graduates.

\section{Student observations:}

These observations originated in discussions and emails with the students regarding the perceived reason for the lack of communication between the two groups in class:

"In Renewable Energy Systems it was split between grad and undergrad. Even in my classes here that tends to be a barrier. Both groups usually perceive the others as a different group. This is partially because they are often different groups. Grad students are often working full time, are older, have families, and are less interested in, for lack of a better term, college shenanigans and more interested in the education"

“... there were enough study abroad students that we didn't NEED to mix. Lots of the people in our group knew each other already and it created a large comfort zone that was easy to stay in. I've noticed this everywhere I've ever gone ... ... The two times when I see this happen less are when you have a very small group, such as our single RIT-NY student this quarter, or when you have students that actively want to go out of their way to meet people."

From these statements it is clear that there is some awareness that an effort is required to establish cross cultural communication, particularly in a large homogeneous group environment, where a "comfort zone" is created. In addition, a perceived barrier is the age difference, and different interests of the students. However, these differing interests can, in addition to the age difference, also be attributed in part to different ethnic backgrounds, and thus bridging them with active communication can significantly enhance global competencies.

\section{Assessment}

The quantitative assessment of the in-class interaction was carried out primarily in the form of a summative survey. The survey was directed at the study abroad students, to gauge their experiences and motivation before, during and after the international experience. In addition, the intention was to measure the depth of the perceived intercultural interaction (primarily in the context of the class), and to compare it with the observations. This is particularly important, as international experiences can be perceived as being very "international”, however adding little to no global competencies. Also the perceived effect of the large visiting group size on the students' willingness to interact was assessed. 


\section{Pre-departure:}

At this stage the specific motivation of the students to visit the study abroad location was probed. The intention was to assess whether there is an underlying interest of the students in the country, as well as a thorough personal preparation in order to maximize the experiences. This selectiveness and preparation can be interpreted as, while not a condition, a strong facilitator for seeking out intercultural experiences (which in turn generate global competencies). The results to the motivation question are summarized in Fig. 1.

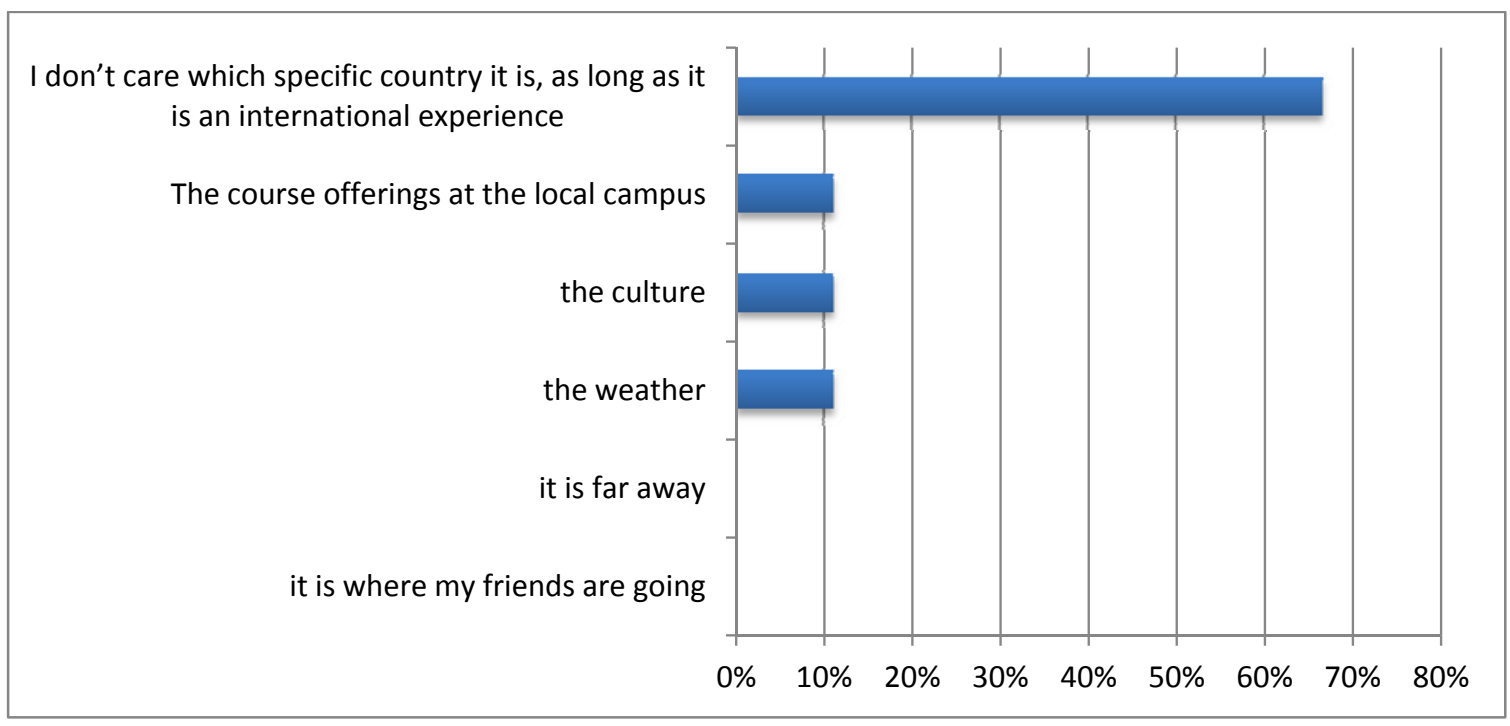

Figure 1. Motivation question: "I want to go to this particular country because ..."

From the above chart it can be seen that there is little interest in the peculiarities of the country. $67 \%$ of the respondents were indifferent about the country, and were only looking for an "international experience".

The scale used in Figures 2 through 5 represents the following statements:

- 4.0: strongly agree,

- 3.0: agree,

- 2.0: indifferent,

- 1.0: disagree,

- 0.0: strongly disagree.

As shown in Figure 2, the majority of the students considered the international experience important for their career. However, the importance of studying and reading up on the host country was not that high, and the overall preparation before departure, in hindsight, did not match the experiences. These results emphasize that, while the more or less abstract concept of “international experience” is deemed important for one’s personal and professional development, the actual depth of this experience, and thus the development of global competencies, is not understood or developed. The concept of becoming more "International" by simply travelling abroad is an apparent misconception. 


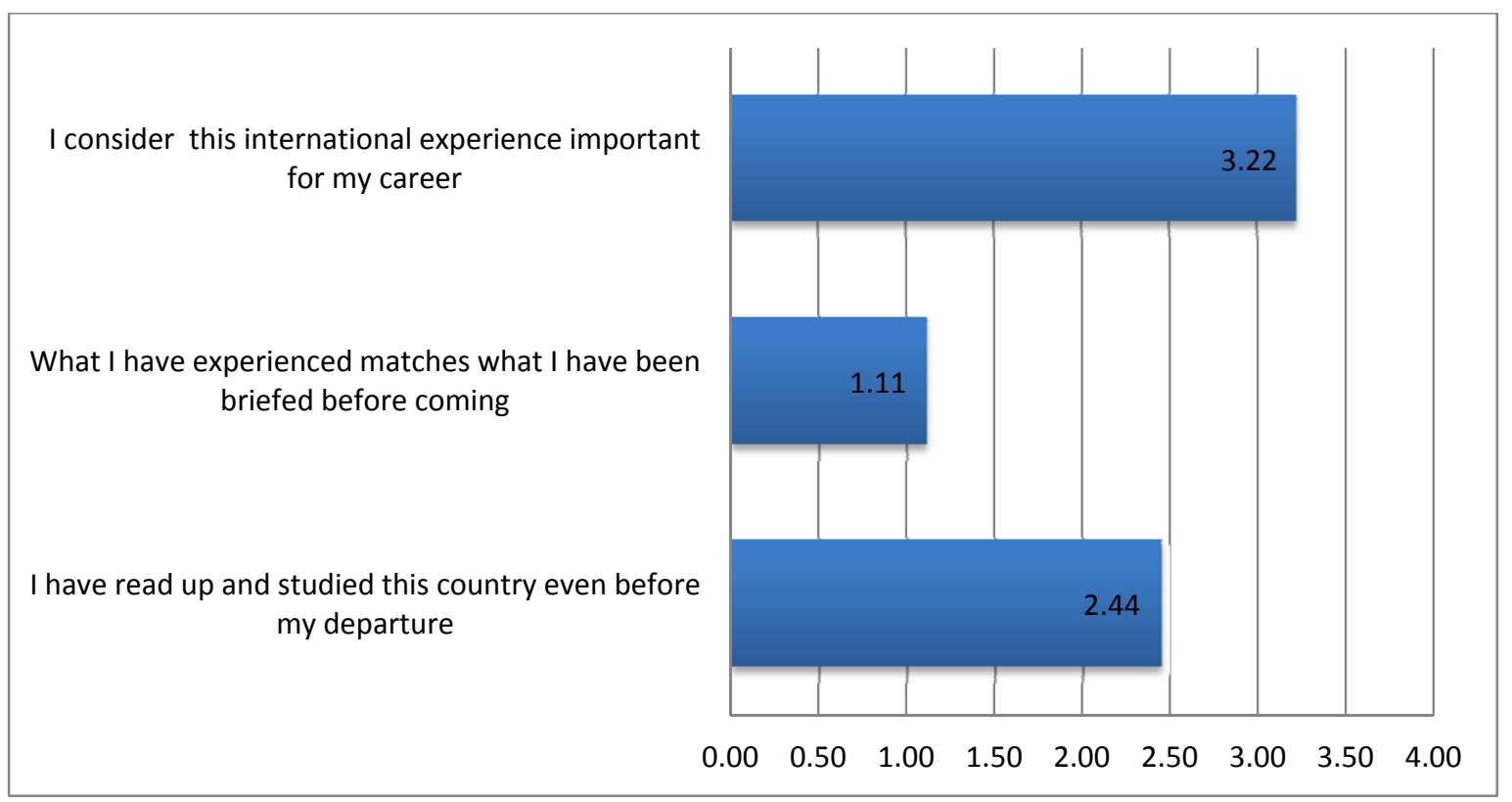

Figure 2. Results from summative survey preparatory portion.

The importance of the home university's preparation in generating the appropriate background to maximize the benefits of the international experience is clear. In addition to providing the academic framework for the students to seamlessly continue their progress towards their degree, the explicit inclusion of global learning objectives and outcomes requires particular focus on enabling the students to develop global competencies. This can only be achieved by educating the students on what it means to attain global competencies and become more world-class engineers. Thus it may be concluded that the pre-departure preparation should cover a twofold purpose:

- The students need detailed information on the environment, procedures and rules to expect. This includes the background knowledge necessary to enable the students to classify their experiences and view them within the appropriate context. For example, if the students are aware of the societal implications of living in an Islamic country, and have the corresponding background knowledge on the origin of these rules, they might be able to comprehend them in a much "deeper” way than if they are simply confronted with them in a uninformed fashion. This in turn facilitates the understanding of differing viewpoints promoting empathy for a different culture, which is at the heart of acquiring global competencies.

- In addition, the preparation should educate the students about global competencies; in particular communication-related issues should be stressed, to enable the students to interact cross-culturally. These interactions are instrumental in developing an ethnorelative standpoint ${ }^{12}$ from an ethnocentric origin.

In the case of this work-in-progress, the pre-briefings provided were viewed as not representing the reality of the experience. This, in combination with the non-inclusion of the global learning outcomes in the course work, generated the group dynamics and associated difficulties of the next phase: 


\section{During the visit}

As discussed previously, strong grouping dynamics were observed in class. The survey explored both the perceived individual interaction levels, as well as the perceived effect of being a part of a larger group.

Over past observations the individual student's level of cross-cultural interaction is to a large extent a function to the availability of same-nationality peers. However, the perceived relationship, as reported in the survey, is weak or inconclusive (Fig. 3):

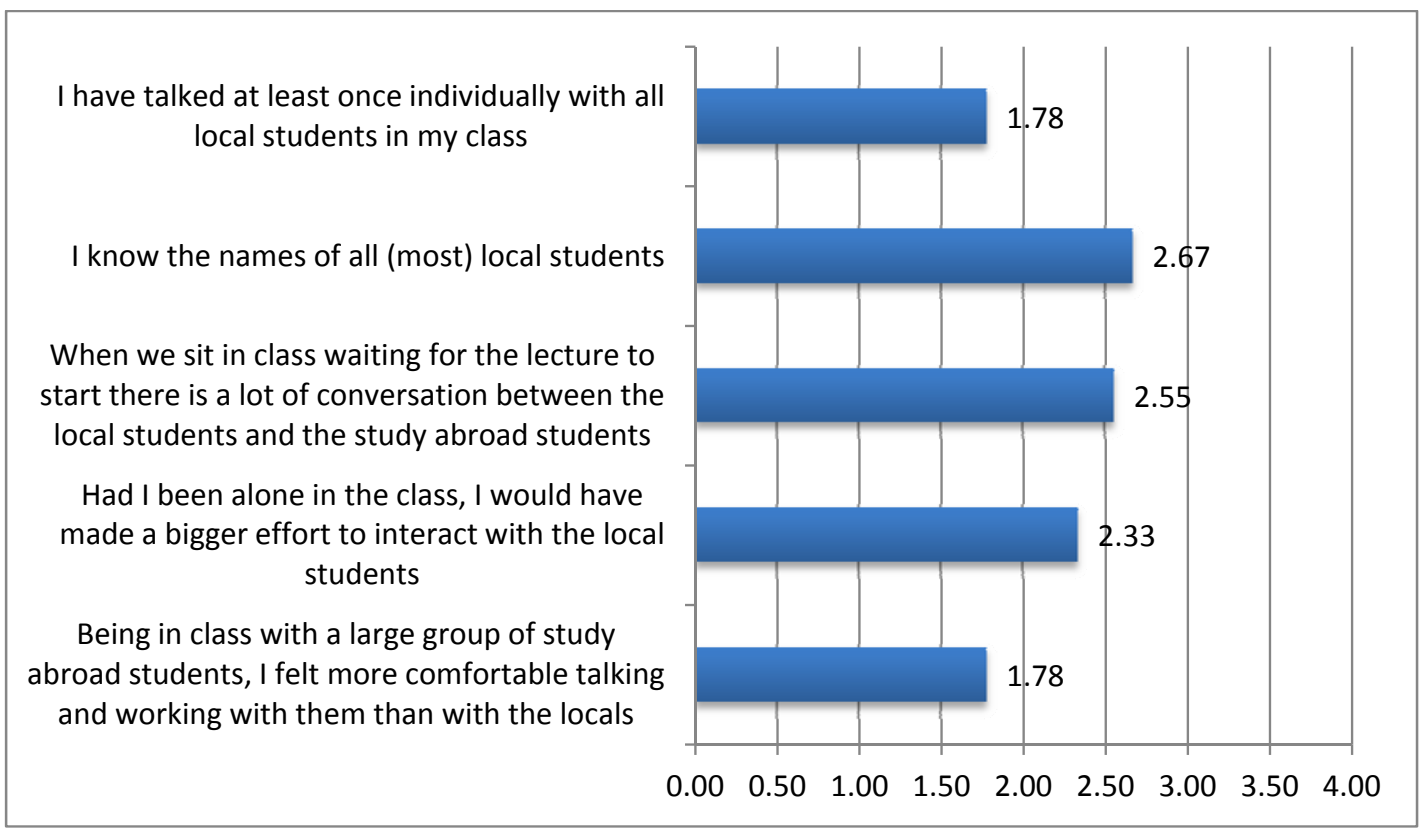

Figure 3. Perceived group dynamics.

As the students report a neutral dependency of their own interaction with the size of their group, and in order for the results to correspond with the class observations, only two possible alternatives develop; one being that the overall need of each individual student to communicate (within either group) is low (this was not observed to be the case, as the level of chatting and communicating within each group were significant), or that the perceived results displayed in Fig. 3 do not correspond well to reality. This may be explained by the bias of the question; in light of the perceived intensity of the international experience (often proportional to distance from home), it is difficult to accept that it is more comfortable to interact with ones own countrymen and women rather than the different cultures of the host country.

This factor becomes more apparent upon inquiring about the favored type of interaction (Fig. 4), where students strongly favor collaborative environments in an evenly split teamwork environment over more individual type interaction. In addition, when asked what their favored type of extracurricular interaction is, $100 \%$ of the students responded that they preferred to participate in activities as part of a group of visiting students, over participating alone. 
As a team of study abroad students with one or two local students to show us the local approach to the question at hand

Alone with a group of local students

In a teamwork environment, split equally between nationalities

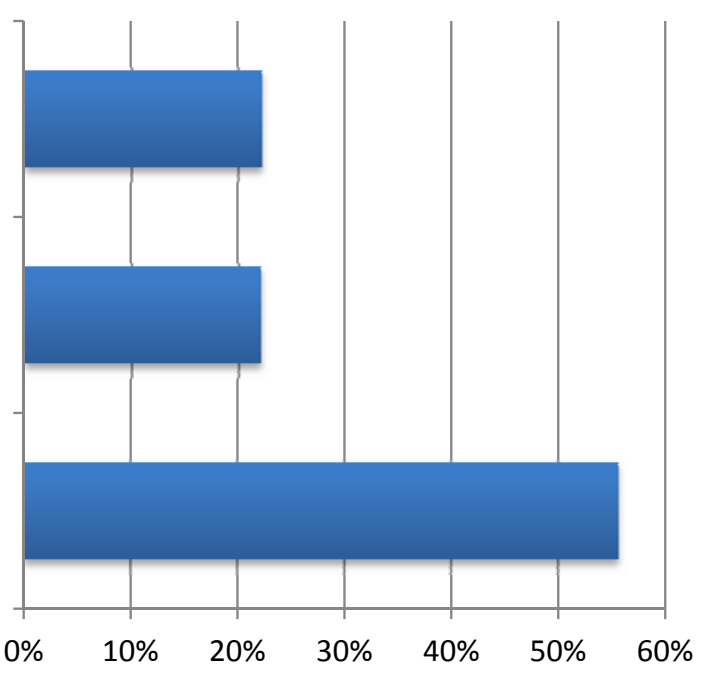

Figure 4. Interaction question: "I prefer to participate in collaborative class activities with the local students ..."

It is clear that students prefer to interact as a group. This type of interaction allows them to stay within their comfort zone, however also transmits a perception of substantial crosscultural interaction. These "safe haven" group dynamics weaken the extent to which global competencies are developed.

\section{After the visit}

This section of the survey addressed the perceived benefits of the experience. Fig. 5 summarized the after visit survey results. In particular the perceived usefulness of the experience towards increased confidence, and the perceived shift to an ethno-relative standpoint was of interest.

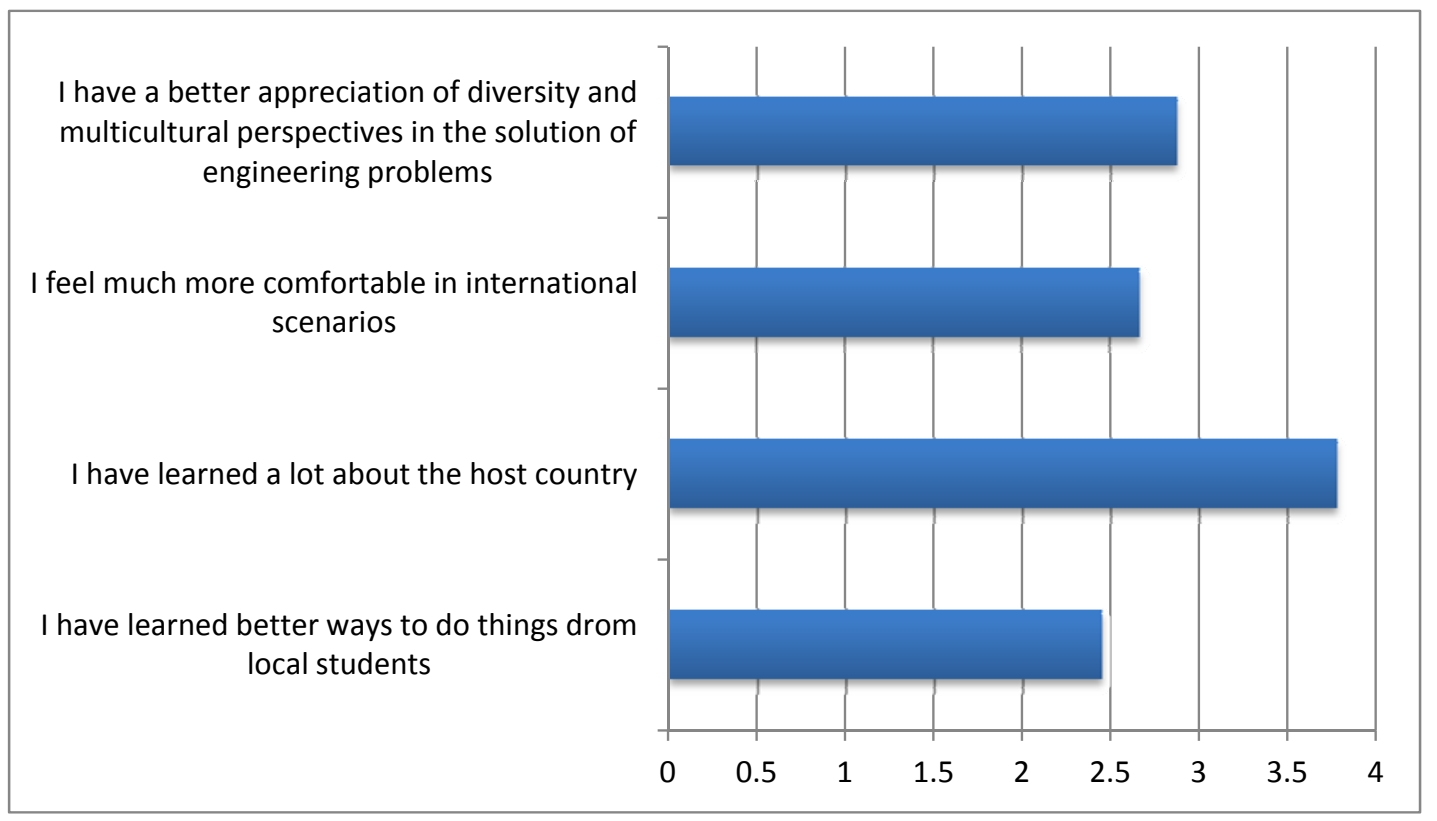

Figure 5. Post-visit survey results. 
The results show an overall positive impression of the experience. The item that displays the highest perceived gain is also the one that requires the lowest amount of global competencies; the simple knowledge-gathering on the host country. Learning about the host country was aided by a number of organized activities, where the group of study abroad students visited the touristic points of interest of the country. Little depth of understanding of the foreign culture is required for this task. However, the indicators of increased global competencies, such as realizing that many things can be learned from other cultures, or that an appreciation for diversity aspects in the context of engineering problems has been developed, show a range of responses between "indifferent" and "agree".

In summary, the students report increased knowledge about the host country, however they do not report a significant increase in cross-cultural understanding. This reinforces the interpretation of the in-class observations; the study abroad experience, while certainly being enriching, does not emphasize the truly important factors to reach a higher level of global competency, but merely offers the benefits of an extensive touristic visit. It is thus important to present and implement a series of goals and measures to enhance the development of the global skills during study abroad experiences, and not simply enhance the students' international exposure.

\section{Global learning outcomes}

The above observations and survey results suggest that a directed effort to increase the depth of the international experience during a study abroad term should be implemented. In particular, the introduction of formal global learning outcomes is suggested at both the program level and the individual course level.

\section{Program level}

At the program level, communication specific global learning outcomes should be introduced. These components, which ideally are introduced in the pre-departure phase, will help the students make the most out of their experience. While the common approach to prepare students for the time abroad is learning about the host country, its culture, history and geography, traditions, etc., (these components constitute an important albeit incomplete

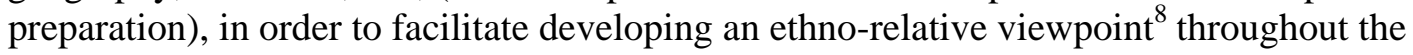
time abroad, significant cross cultural communication skills need to be acquired and their application stimulated. Introducing specific pre-departure workshops and briefings to enhance cross cultural communication skills (in addition to the traditional country specific orientation) will stimulate curiosity about the similarities and differences between the cultures, and provide tools to interact and reflect on the interaction in a confident and constructive way. In addition to this pre-departure preparation, and in the context of the formative evaluation of the effectiveness of these components, some specific (and non-course related) tasks can be introduced and scheduled throughout the time abroad, for example initiating and executing discussion rounds, writing a paper on a cross culturally sensitive topic with a local student, etc. Some communication specific outcomes introduced at this level may include:

- Learn through listening and observing ${ }^{12}$

- Establish rapport with people of different cultures; communicate despite barriers.

- Sustain difficult conversations in the face of highly emotional and perhaps uncongenial differences ${ }^{13}$. 
The evaluation of these program level components can be achieved through quantitative formative assessment of the scheduled tasks and exercises, as well as summative survey type reflection on perceived development of the students' global skills, such as the survey conducted in the context of this work or described in the literature ${ }^{14}$. In addition, an effort should be made to increase awareness of the individual course-instructors towards fostering cross-cultural communication, and to provide feedback on their observations on the effect of the measures and the evolution of the students. In a summative manner these observations should then be compared again with the students' perceptions, and the process iterated.

\section{Individual course level}

The development of global competency in the course environment will benefit significantly from having introduced the program scale objectives and outcomes in a pre-departure phase. Students within the individual course should have been briefed on displaying a greater effort to communicating interculturally. However, in addition to profiting from this propensity, which is particularly beneficial in the large grouping environment addressed here, each specific course should incorporate individual outcomes to reinforce the overall global competencies. This naturally requires an expansion of the normal course learning outcomes to account for the development of these skills.

- Able to work effectively as a part of a multinational/multicultural team ${ }^{12}$

- Effective and knowledgeable in working in cross-cultural settings ${ }^{12}$

These outcomes should be assessed and become an integral part of the course grade.

\section{Conclusions}

Study abroad programs offer an ideal environment not only to provide international experiences for students, but also to develop global awareness and global competencies. While international experience can be acquired by simple touristic activities, the development of global skills requires an additional interest and immersion in another culture to gain a depth understanding of cultural differences and develop empathy for other cultures. In order to foster the development of these skills, a focus on cross cultural communication and activities needs to be instilled as a fixed component of the international experience.

In assessing the overall experience, it can be concluded that students perceive their level of interaction to be more than what is observed. This is due to two factors: one is the perceived strength of the international experience (which as it was said before is often perceived proportional to the distance travelled), and the other being the dynamics of homogeneous groups travelling together; the cultural interaction often happens at the group level, which weakens the individual exposure, but is perceived as intense.

Even though there is a perception that learning might occur simply by exposing the students to certain practices (learning by doing approach), it is observed that in the case of global competencies there should be a mindset preparation for the learning experience to happen. For example, the tendency of US students to believe that no other language is necessary or what is right here must be right everywhere, do not help to develop an open mindset to accept cultural difference and, therefore, learning of global competencies will not occur. 
The use of a study abroad program as an effective means to foster global competencies requires preparing the students prior to the trip to open their mindset, and get them immersed in the culture during the trip to appreciate the cultural differences and develop the global skills. The following actions are recommended:

- Pre trip preparation:

o Communicate global learning objectives

o Ask students to learn about the culture and heritage of the host country (history, geography, traditions, language, food, dress style, entertainment, music, social behavior, politeness, demonstration of friendship)

o Open students' mindset for a global experience (developing global skills is more than just travelling abroad)

- Visit expectation:

o Students get immersed in the culture (get depth understanding of cultural differences going beyond the obvious differences, develop empathy for different culture, understand and accept the way of thinking of international peers)

o Students develop cross-cultural communication (work in multinational teams, put in practice your understanding of cultural differences to effectively work and interact with your international partners, open your mind and recognize any cultural barriers that might make difficult the communication)

\section{Acknowledgements:}

The authors wish to thank RIT and RIT Dubai for providing the international opportunities for their students that constitute the basis for this research, and specially to the students that have participated in the program, who have collaborated intensively in better understanding and improving the experience.

\section{References}

1. Criteria for accrediting Engineering Programs: Effective for Reviews during the 2012-2013 Accreditation Cycle. http://www.abet.org/

2. National Academies of Engineering of the National Academies, The Engineer of 2020: visions of engineering in the new century, National Academies Press, Washington, DC, 2004.

3. National Academies of Engineering of the National Academies Educating the engineer of 2020: adapting engineering education to the new century, National Academies Press, Washington, DC, 2005.

4. Rising above the Gathering Storm: Energizing and Employing America for a Brighter Economic Future, National Academies Press, Washington, DC, Pre-Publication Version February 2006. http://darwin.nap.edu/books/0309100399/html/R1.html

5. In Search of Global Engineering Excellence: Educating the next generation of engineers for the global workplace. Technische Universität Darmstadt, Germany; Eidgenössische Technische Hochschule Zürich, Switzerland; Georgia Institute of Technology, USA; Massachusetts Institute of Technology, USA; Shanghai Jiao Tong University, China; Tsinghua University, China; Universidade de São Paulo, Brazil; University of Tokyo, Japan; Continental AG, Germany, 2007. http://www.global-engineeringexcellence.org.

6. Esparragoza, I.E., Larrondo-Petrie, M., Jordan, R., and Paez, J. Forming the Global Engineer for the Americas: Global Educational Experiences and Opportunities Involving Latin America and the Caribbean. Proceeding of the 2007 ASEE Annual Conference and Exhibition, Honolulu, Hawaii June 24 to 27, 2007, AC 2007-576.

7. Esparragoza, I.E., Larrondo-Petrie, M., and Friess, W. A. Developing Assessment Tools for International Experiences in Engineering Education. Proceeding of the 2008 ASEE Annual Conference and Exhibition, Pittsburgh, PA June 22 to 25, 2008. AC 2008-656. 
8. Demetry, C. Work in Progress - Development of Intercultural Sensitivity from Study Abroad Programs. Proceeding of the 2007 ASEE/IEEE Frontiers in Education Conference, Milwaukee, October 10-13 2007.

9. Warnick, G.M., Global Competence: Its Importance for Engineers Working in a Global Environment. Proceeding of the 2011 ASEE Annual Conference and Exhibition, Vancouver, Canada June 26 to 29, 2011. AC 2011-350.

10. Friess, W.A., A sustainable buildings course for an international student population; enhancing understanding of geographic and seasonal factors through energy simulation, Proceedings of the WEE2011 SEFI Meeting (ASEE Track), Lisbon, Portugal, September 27-30 2011.

11. http://cindyking.biz/cross-cultural-skills/

12. Language Corps, Jed Willard. Global Competency. Accessed online www.LanguageCorps.com Dec 11 2010.

13. Hovland, K. Global Learning: aligning Student Learning Outcomes with Study Abroad. Association of American Colleges and Universities, NAFSA May 2010. Accessed online http://dev.nafsa.org/uploadedFiles/NAFSA_Home/Resource_Library_Assets/Networks/CCB/AligningLearni ngOutcomes.pdf Dec. 102011.

14. Cutler, S. Borrego, M.. Developing Global Competence in Graduate Engineering and Science Students through an IGERT International Internship Program. Proceedings of the $40^{\text {th }}$ ASEE/IEEE Frontiers in Education Conference, Washington DC, October 27-30 2010. 